\title{
Does Bronchoscopic Lung Volume Reduction Reduce Mortality in Patients with Severe Emphysema?
}

\author{
Demet Turan, Deniz Dogan, Mustafa Cortuk, Elif Tanriverdi, Mehmet Akif Ozgul and Erdogan Cetinkaya \\ Department of Pulmonology, Yedikule Pulmonary Diseases and Thoracic Surgery Education and Research Hospital, Turkey
}

\begin{abstract}
Objective: To compare the 12-month mortality for patients with severe emphysema who underwent either endobronchial valve (EBV) or coil treatments with those managed with standard of care (SoC). Bronchoscopic lung volume reduction (BLVR) is a useful treatment option in patients with chronic obstructive pulmonary disease (COPD), who have severe emphysema.

Study Design: A case-control study.

Place and Duration of Study: Department of Pulmonology, Yedikule Pulmonary Diseases and Thoracic Surgery Education and Research Hospital, Turkey, between January 2018 and January 2019.

Methodology: Medical data of patients diagnosed with severe/very severe emphysema between January 2010 and January 2017 were evaluated. One hundred and forty-eight patients with advanced COPD-emphysema phenotype, who met the BLVR treatment criteria, were evaluated. One hundred and twenty-four patients with 12-month follow-up data, 73 patients treated with BLVR, 43 cases of EBV, 30 cases of coil treatment, and 51 patients managed with standard of care (SoC) were analysed for this study.

Results: A total of $20(16.1 \%)$ patients died at the end of 12 th month and $4(3.2 \%)$ in the early period. At the end of the 12th month, mortality was found in 7 patients $(9.6 \%)$ in the BLVR group ( 3 underwent EBV and 4 received coil treatment, respectively), and $13(25.5 \%)$ patients in the SoC group. There was no statistically significant difference in mortality between groups in the early period, but it was lower in the BLVR group at the end of 12th month.
\end{abstract}

Conclusion: BLVR treatment significantly decreases mortality compared to SoC in patients with advanced emphysema.

Key Words: Emphysema, Mortality, Endobronchial valve, Coil, Bronchoscopic lung volume reduction.

How to cite this article: Turan D, Dogan D, Cortuk M, Tanriverdi E, Ozgul MA, Cetinkaya E. Does Bronchoscopic Lung Volume Reduction Reduce Mortality in Patients with Severe Emphysema?. J Coll Physicians Surg Pak 2021; 31(01):60-64.

\section{INTRODUCTION}

Chronic obstructive pulmonary disease (COPD) is the fourth most common cause of death in the world, and its rate is increasing day by day. ${ }^{1}$ Emphysema, which is a component of COPD, is a disease characterised by progressive and irreversible destruction of the alveolar tissue. The reduction of the elastic retraction, as a result of this destruction, leads to increased air trapping, which is the most important factor in the resulting dyspnea and decrease in the exercise capacity and quality of life. ${ }^{2}$

At present, standard treatment options for patients with COPD include smoking cessation, bronchodilation, mucolytic agents, phosphodiesterase 4 inhibitors, pulmonary rehabilitation, nutritional support, vaccination against influenza, pneumococcus, and long-term oxygen therapy. ${ }^{3}$

Correspondence to: Dr. Demet Turan, Department of Pulmonology, Yedikule Pulmonary Diseases and Thoracic Surgery Education and Research Hospital, Turkey

E-mail: drdemetturan@gmail.com

Received: June 26, 2020; Revised: December 08, 2020;

Accepted: December 22, 2020

DOI: https://doi.org/10.29271/jcpsp.2021.01.60
Although these treatments help increase the exercise capacity of patients and decrease symptom exacerbation, they do not cure the pathophysiology of the disease or prevent disease progression.

Lung volume reduction surgery was considered a light of hope, but the high mortality rates observed in the early postoperative period were disappointing. ${ }^{4}$ Alternatively, the successful and promising results of BLVR treatments at the beginning of this century, brought a new dimension to the treatment of emphysema. ${ }^{5}$ BLVR treatments, including endobronchial valve (EBV), ${ }^{5}$ coil, ${ }^{6}$ and bronchoscopic thermal vapour ablation (BTVA), ${ }^{7}$ are the most commonly applied methods among the treatmentoptions.

The aim of the treatment of COPD is to improve the shortness of breath, exercise capacity, and quality of life, ultimately increasing the patient's life expectancy and preventing death related to COPD.

In this study, the aim was to compare the mortality between the BLVR and the SoC groups in the early period and at the end of the $12^{\text {th }}$ month in patients with severe emphysema.

\section{METHODOLOGY}

This retrospective case-control study was conducted at the 
Department of Pulmonology, Yedikule Pulmonary Diseases and Thoracic Surgery Education and Research Hospital, Turkey, between January 2018 and January 2019. Medical data of patients diagnosed with severe / very severe emphysema between January 2010 and January 2017 were evaluated. The study was performed in a single centre.

The patients were divided into case (treated with BLVR) and control (unable to undergo BLVR due to difficulties in obtaining BLVR equipment and materials) groups. Early (first six weeks) and 12-month mortality were assessed. The protocol was approved by the Ethical Committee of the Faculty of Medicine of Karabuk University (Prot. 1/31).

The study population consisted of patients who underwent pulmonary rehabilitation (PR) for at least four weeks before the BLVR treatment plan, and used optimal medical treatment according to the GOLD guidelines. ${ }^{3}$

The following criteria were used for eligibility for inclusion for BLVR procedures: an age of $40-75$ years, $\mathrm{FEV}_{1} 15 \%-45 \%$ of predicted value, $\mathrm{RV} \geq 150 \%$ of predicted value ( $\geq 200 \%$ for coil treatment), $\mathrm{TLC}>100 \%, \mathrm{PaCO}_{2}<50 \mathrm{mmHg}, \mathrm{PaO}_{2}>45 \mathrm{mmHg}$ (in room air), DLCO $>20 \%$ of predicted value, six-minute walking test of 150-400 meters, sPAP $<50 \mathrm{mmHg}$ in echocardiography, using optimal bronchodilator treatment, non-smoking for at least eight weeks prior to the procedure, having a PR at least four weeks prior to the procedure, and not using any anti-coagulant or anti-platelet treatment (for coil treatment). The other group consisted of patients who received optimum medical treatment and were eligible for BLVR treatment, but could not undergo the procedure due to difficulties in obtaining BLVR equipment and materials. This group of patients formed the medical treatment group of this study, medical treatment group (SoCgroup) of this study.

Inclusion and exclusion criteria were determined according to the NETT study and Slebos's study.,

All EBV insertion procedures were performed under conscious sedation (2-5 mg midazolam) by a flexible bronchoscope (Olympus BF-1TQ180) with 2.8 mm working channel. Quantitative lung perfusion scintigraphy data and thin-section HRCT examination were used in the selection of the target lung lobe. Collateral ventilation was assessed with the Chartis system (Pulmonx Corporation, Redwood City, CA, USA) before the valve placement procedures; only patients with no collateral ventilation in the target lobe were included. Appropriately sized valves (Zephyr ${ }^{\circledR}$ EBV; Pulmonx Corporation, Redwood, CA, USA) were placed in all segments or subsegments of the target lung lobe. Chest X-rays were taken in all patients suspected of pneumothorax immediately after the procedure, and two hours after the procedure, even if there was no complaint. Patients who had no complications in the early postprocedure period were discharged after an average of six hours of monitoring and were called for control after 24 hours and one week.

All coil procedures were performed under general anesthesia. In these patients, quantitative lung perfusion scintigraphy data and thin-section HRCT examination were used in the selection of the lung lobe for the coil to be placed. The bronchial system of the target lung lobe in the intubated patient was evaluated, and the appropriate-length coils (RePneu LVR coil, PneumRx, USA) were placed in the subsegments of the lobe about $2 \mathrm{~cm}$ from the pleura under fluoroscopy guidance. Chest X-ray was performed for the evaluation of pneumothorax immediately in patients with complaints, and at the $2^{\text {nd }}$ hour after the procedure routinely in patients without complaints. After the procedure, all patients were hospitalised for 3-5 days and treated with systemic steroids for one week. Patients who had no complication in the early postprocedure period were discharged and were called for control one week after the procedure.

Patients, who underwent BLVR treatment, were assesed at the 7 th and 28 th days and every three months. Appropriate treatments according to the GOLD guideline3 were given to patients managed with SoC, and their controls were made in quarterly periods. Decedents during the six weeks were described as early mortality. Patients who could not complete a 12-month follow-up period for any reason were excluded from the study.

SPSS for Mac 20.0 package programme (SPSS Inc, Chicago, IL) was used for the statistical evaluation. Data were summarised as the mean and standard deviation for the continuous variables and as the absolute value and percentages for the categorical variables. Chi-square test/Fisher's exact test for the categorical variables and Student's t-test were used for the continuous variables. Ap-value of less than 0.05 was considered to be significant with a $95 \%$ confidence interval.

\section{RESULTS}

One hundred and forty-eight patients, who met the BLVR treatment criteria, were evaluated retrospectively. Of these, 76 patients had received BLVR treatment; and the remaining 72 patients had not undergone a BLVR procedure and were included in the SoC group. Forty-six of the 76 BLVR patients had been treated with EBV and 30 had been treated with coils. Three patients in the BLVR group and 21 patients in the SoC group were excluded from the study. In the BLVR group, one patient with all valves removed on the $124^{\text {th }}$ day after the procedure was excluded, and the 12-month follow-up period was not completed yet in two patients. In the SoC group, 16 patients were excluded from the study because they did not come to follow-ups, and the 12-month follow-up period was not yet completed in five patients. The data of the rest of the 124 patients were included in the study: 73 received BLVR treatment ( 43 patients received EBV and 30 coil treatment) and 51 were managed with SoC (Figure 1).

Demographic and clinical features, comorbidities, and mortality rates were compared between the two groups. Correlatively, GOLD stages (III/IV) in the BLVR and SoC treatment groups were $27 / 46$ versus $25 / 26$, respectively; and there was no significant difference $(p=0.181)$. Baseline demographic data, comorbidities, pulmonary functions, and exercise performances of the groups weresimilar(TableI). Cardiovasculardiseases and hypertension were the most common comorbidities. 
Table I: Baseline characteristics of the patients.

\begin{tabular}{|c|c|c|c|}
\hline Variable & $\begin{array}{l}\text { BVLR Group } \\
(n=73)\end{array}$ & $\begin{array}{l}\text { SoC Group } \\
(n=51)\end{array}$ & p-value \\
\hline \multicolumn{4}{|c|}{ Demographic characteristic } \\
\hline Age, years & $63.1 \pm 7.5$ & $62.5 \pm 8.8$ & 0.713 \\
\hline Female / male & $8(11 \%) / 65(89 \%)$ & $1(2 \%) / 50(98 \%)$ & 0.080 \\
\hline Pack year smoking & $50.0 \pm 30.4$ & $56.7 \pm 36.3$ & 0.313 \\
\hline Comorbidities & $54(\overline{74} \%)$ & $37(7 \overline{2} .5 \%)$ & 0.860 \\
\hline \multicolumn{4}{|l|}{ Clinical characteristics } \\
\hline GOLD stage (III/IV) & $27(37 \%) / 46(63 \%)$ & $25(49 \%) / 26(51 \%)$ & 0.181 \\
\hline \multicolumn{4}{|l|}{ Lung function } \\
\hline TLC, L & $8.08 \pm 1.86$ & $8.08 \pm 1.83$ & 0.992 \\
\hline TLC, \% predicted & $132.5 \pm 30.7$ & $132.0 \pm 25.9$ & 0.930 \\
\hline$R V, L$ & $5.59 \pm 1.87$ & $5.59 \pm 1.76$ & 0.995 \\
\hline RV, \% predicted & $246.9 \pm 81.3$ & $243.6 \pm 67.1$ & 0.815 \\
\hline RV/TLC, \% & $67.2 \pm 12.9$ & $68.8 \pm 6.9$ & 0.458 \\
\hline FVC, L & $2.30 \pm 0.62$ & $2.29 \pm 0.58$ & 0.933 \\
\hline FVC, \% predicted & $65.2 \pm 18.6$ & $63.7 \pm 12.4$ & 0.618 \\
\hline FEV1, L & $0.78 \pm 0.20$ & $0.84 \pm 0.23$ & 0.097 \\
\hline FEV1, \% predicted & $27.9+7.8$ & $30.2 \pm 7.6$ & 0.104 \\
\hline $\mathrm{DLCO}, \mathrm{mmol} / \mathrm{min} / \mathrm{kPa}$ & $10.98 \pm 5.42$ & $11.21 \pm 4.17$ & 0.852 \\
\hline DLCO, \% predicted & $44.0 \pm 20.5$ & $44.6 \pm 15.4$ & 0.903 \\
\hline $\begin{array}{l}\text { Exercise performance } \\
6 M W D, m\end{array}$ & $260 \pm 119.9$ & $275.9 \pm 104.5$ & 0.514 \\
\hline
\end{tabular}

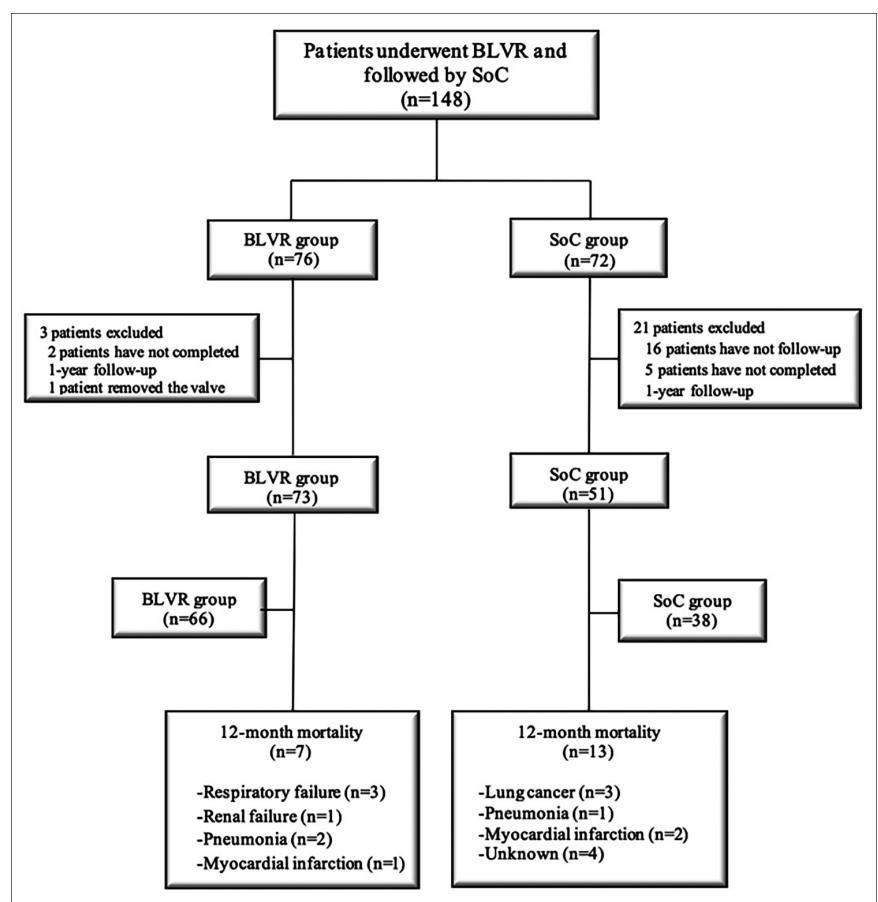

Figure I: The flow chart of the study.

When the mortality rates of the whole study population were examined, it was seen that mortality occurred in 20 patients (16.1\%), in which 12-month follow-up period was completed, and the rate of early period mortality (first six weeks) was found to be $3.2 \%$ (4 cases). A total of seven patients (9.6\%) died in the BLVR treatment group. Of these, three patients had EBV, and four patients were treated with coils. In the BLVR group, the early period mortality occurred in a total of two patients (2.7\%): one of the patients had EBV and the other had coil treatments. In the EBV-treated patients, one patient died due to end-stage respiratory failure on the $7^{\text {th }}$ day after the procedure, one died on the $145^{\text {th }}$ day due to end-stage renal failure, and one died on the $147^{\text {th }}$ day due to pneumonia developing at the distal end of the valve. In the coil-treated patients, one patient each died due to hemoptysis on the $8^{\text {th }}$ day after the procedure, end-stage respiratory failure on the $21^{\text {st }}$ day, myocardial infarction on the $83^{\text {rd }}$ day, one died due to end-stage respiratory failure on the $186^{\text {th }}$ day; and in the SoC group, mortality at the end of 12 th month was observed in a total of 13 patients $(25.5 \%)$, with two deaths (4\%) in the first six weeks. Most of the causes of mortality were pulmonary, which included end-stage respiratory failure $(n=3)$, lung cancer $(n=3)$, pneumonia $(n=1)$, and cardiovascular causes $(n=2)$. The cause of death was unclear in four cases.

It was seen that mortality rate was significantly lower in patients who underwent BLVR treatment when compared with the SoC group ( $n=7,9.6 \%$, vs. $n=13,25.5 \%$, respectively) at the end of the first year $(p=0.018)$. When subgroup analysis was performed in the BLVR treatment group, the mortality rate was lower in the EBV group; but it was not statistically significant $(n=3,7 \%$, in EBV vs. $n=4$, $13.3 \%$, in the coil, $p=0.435$ ). It was also determined that all of the decedent patients in the BLVR group were at GOLD stage IV. Alternatively, the mortality rate in patients at GOLD stages III and IV was similar $(\mathrm{n}=7,28.0 \%$, vs. $\mathrm{n}=6$, $23.04 \%$ ) in the SoC treatment group.

\section{DISCUSSION}

Emphysema is one of the leading causes of disability worldwide, despite the advancements in medical treatment and PR. BLVR treatments are a promising method with positive results for patients with severe emphysema. ${ }^{5,8,9}$ 
This study showed that although early mortality rates were similar between BLVR and SoC groups, mortality was significantly lower in patients who underwent BLVR $(9.6 \%)$ at the end of the 12th month. In other words, mortality was significantly higher at the end of the first year in patients who were managed by SoC, with a mortality rate of $25.59 \%$ in this group. To the best of authors knowledge, this is the first study in which the BLVR and SoC groups were evaluated together for mortality.

As in many other studies on EBV or coil treatments, demographic data, pulmonary functions, and exercise performances were similar between pre-treatment demographic data, pulmonary functions, and exercise performances were similar between these patient groups. ${ }^{9-12}$ In the VENUTA study, 33 patients were able to be followed up for a mean of 32 months; mortality rate was $40 \%$. Mortality was not related to the procedure, and the most frequent cause of death was lung cancer and end-stage respiratory failure. ${ }^{13}$ In this study, three out of 43 patients, who underwent EBV after 12 months, had mortality. The causes of death in these cases were end-stage respiratory failure, renal failure, and obstructive pneumonia. The presently reported mortality rate was significantly lower than VENUTA study, which, we think, may be due to the short follow-up in this study.

In the VENT study, 220 patients underwent EBV treatment, and 101 patients were managed with SoC. The mortality rates in this study were similar between the two groups after 12 months of follow-up ( $3.7 \%$ vs. $3.5 \%$, respectively). ${ }^{5}$ In the European section of the same study, mortality was observed in a total of 10 patients after a one-year follow-up; four (7\%) of these patients were in the EBV treatment group, while 6 (5\%) were in the SoC group. At the same time, no procedure-related deaths were seen in this study. ${ }^{14}$ Although the mortality rate and follow-up period in these studies were the same as in the EBV group of this study; in this study, the total mortality was lower in the BLVR group.

In the Renew study, 158 patients underwent coil treatment, while 157 were managed with SoC, including PR. ${ }^{12}$ Researchers in this study reported that mortality was observed in 10 patients $(6.5 \%)$ in the coil treatment group and eight $(5.1 \%)$ patients in the SoC group in the first year. This result was not statistically significant. In the Revolens study, ${ }^{15}$ comparing coil treatment and SoC, 100 patients (71 males, 29 females) were randomised into two equal groups. As a result of this study, a total of seven deaths were reported at the end of $12^{\text {th }}$ month: four in the SoC group and three in coil therapy group in PR group. In this study, only one death related to the procedure was observed; other deaths occurred due to pulmonary causes.

In this study, patients in the BLVR and SoC groups showed homogeneous features in terms of demographic characteristics and comorbidities. However, unlike the literature, mortality rates were significantly lower in the BLVR group compared with the SoC group in the present study. Previously published studies are mostly prospective. ${ }^{5,9,12-17}$ Since patients included in prospective studies are more strictly followed, any complication, exacerbation, or side effect is immediately detected and treated accordingly, thus lowering mortality rates. Medically treated patients may suffer from side effects or excacerbations without referring to a medical professional or may simply skip their follow-up appointments. The authors believe that the low mortality rate in the BLVR group in this study was related to this situation.

In a retrospective study by Trudzinski et al., 20 patients with FEV1 values of $\leq 20 \%$ were evaluated, and they reported that EBV treatment was applicable and safe in terms of 30-day mortality.$^{18}$ In another study conducted by Darwiche et al., the data of 20 patients with FEV $1 \leq 20 \%$ were also evaluated, and EBV treatment was reported as feasible and safe. ${ }^{19}$ In another retrospective study conducted by Simon et al., 33 patients with FEV1 $\leq 20 \%$ received coil treatment, and there was no respiratory failure or death requiring mechanical ventilation in the first three months. ${ }^{20}$ In the present study, all seven patients with mortality in the BLVR group were at GOLD stage IV. There was no significant difference between patients at GOLD stages III and IV in the SoC group. Although these three publications indicated that BLVR application was safe in patients with severe emphysema having severe respiratory restriction (FEV1 $\leq 20 \%$ ), due to the short follow-up period and low number of cases, new studies are needed for evaluating BLVR treatment in this patient group.

This study has limitations. First, it is a retrospective data. Second, it has relatively a small number of cases. However, it is real-life data making it very relevant.

\section{CONCLUSION}

Use of BLVR in patients with severe COPD-emphysema may reduce mortality in well-selected patients compared with SoC treatment at the end of 12 th month. Patients at GOLD stage IV should be followed up more closely after BLVR because of higher chances of death.

\section{ETHICAL APPROVAL:}

The protocol was approved by the Ethical Committee of the Faculty of Medicine of Karabuk University (Prot. 1/31).

\section{PATIENTS' CONSENT:}

It is a retrospective study, informed consent forms were not received from the patients.

\section{CONFLICT OF INTEREST:}

All authors declared no conflict of interest.

\section{AUTHORS' CONTRIBUTION:}

DT, EC: Designed research / study, collected data, authored the paper.

DD, MC: Performed research / study, analysed data, authored the paper. 
MAO, ET: Contributed important reagents.

\section{REFERENCES}

1. Harb N, Foster JM, Dobler CC. Patient-perceived treatment burden of chronic obstructive pulmonary disease. Int J Chron Obstruct Pulmon Dis 2017; 12:1641-52. doi: 10.2147/COPD.S130353.

2. The American Thoracic Society Standards for the diagnosis and care of patients with the chronic obstructive pulmonary disease. Am J Respir Crit Care Med 1995; 152: p77-121.

3. Global Initiative for Chronic Obstructive Lung Disease. Global strategy for the diagnosis, management, and prevention of chronic obstructive pulmonary disease. 2017. Access: http://www.goldcopd.org/

4. Weinmann GG, Chiang YP, Sheingold S. The national emphysema treatment trial (NETT). A study in agency collaboration. Proc Am Thorac Soc 2008; 5(4):381-4. doi: 10.1513/pats.200709-154ET.

5. Sciurba FC, Ernst A, Herth FJF, Strange C, Criner GJ, Marquette $\mathrm{CH}$, et al. A randomised study of endobronchial valves for advanced emphysema. N Engl J Med 2010; 363(13):1233-44. doi: 10.1056/NEJMoa0900928.

6. Shah PL, Zoumot Z, Singh S, Bicknell S, Ross ET, Quiring J, et al. Endobronchial coils for the treatment of severe emphysema with hyperinfl ation (RESET): A randomised controlled trial. Lancet Respir Med 2013; 1(3):233-40. doi: 10.1016/ S2213-2600(13)70047-X.

7. Gompelmann D, Eberhardt R, Schuhmann M, Valipour A, Shah PL, Herth FJ, et al. Lung volume reduction with vapor ablation in the presence of incomplete fissures: 12-month results from the step-up randomised controlled study. Respiration 2016; 92(6): 397-403. doi: 10.1159/000452424

8. Slebos DJ, Klooster K, Ernst A, Herth FJF, Kerstjens HAM. Bronchoscopic lung volume reduction coil treatment of patients with severe heterogeneous emphysema. Chest 2012; 142(3):574-82. doi: 10.1378/chest.11-0730.

9. Kemp SV, Slebos DJ, Kirk A, Kornaszewska M, Carron K, Ek L, et al. a multicenter randomised controlled trial of zephyr endobronchial valve treatment in heterogeneous emphysema (transform). Am J Respir Crit Care Med 2017; 196 (12):1535-43. doi: 10.1164/rccm.201707-13270C.

10. Davey C, Zoumot Z, Jordan S, McNulty WH, Carr DH, Hind $M D$, et al. Bronchoscopic lung volüme reduction with endobronchial valves for patients with heterogeneous emphysema and intact interlobar fissures (the BeLieVeR- HIFi study): A randomised controlled trial. Lancet 2015;
386(9998):1066-73. doi: 10.1016/S0140-6736(15)60001-0.

11. Valipour A, Slebos DJ, Herth F, Darwiche K, Wagner M, Ficker $\mathrm{JH}$, et al. Endobronchial valve therapy in patients with homogeneous emphysema: Results from the IMPACT study. Am J Respir Crit Care Med 2016; 194(9):1073-82. doi: 10.1164/rccm.201607-13830C.

12. Sciurba FC, Criner GJ, Strange C, Shah PL, Michaud G, Connolly TA, et al. Study research group. Effect of endobronchial coils vs usual care on exercise tolerance in patients with severe emphysema.The Renew Randomised Clinical Trial. 2016; 315(20):2178-89. doi:10.1001/ jama. 2016.6261

13. Venuta F, Anile M, Diso D, Carillo C, Giacomo T, D’Andrilli A, et al. Long term follow up after bronchoscopic lung volüme reduction in patients with emphysema. ERJ Express 2011; 39(5):1084-9. doi: 10.1183/09031936.00071311.

14. Herth FJF, Noppen M, Valipour A, Leroy S, Vergnon J, Ficker $\mathrm{JH}$, et al. İnternational VENT Study Group. Efficacy predictors of lung volume reduction with zephyr valves in a European cohort. Eur Respir J 2012; 39(6): 1334-42. doi: 10.1183/ 09031936.00161611.

15. Desle G, Mal H, Dutau H, Bourdin A, Vergnon JM, Pison C, et al. Lung volume reduction coil treatment vs usual care in patients with severe emphysema the revolens randomised clinical trial. JAMA 2016; 315(2):175-84. doi: 10.1001/ jama.2015.17821.

16. Criner GJ, Sue R, Wright S, Dransfield M, Perez HR, Wiese T, et al. A multicenter rct of zephyr ${ }^{\circledR}$ endobronchial valve treatment in heterogeneous emphysema (LIBERATE). AJRCCM Articles in Press 2018; 198(9):1151-64. doi: 10.1164/rccm.201803-05900C.

17. Klooster K, Hacken NHT, Hartman JE, Kerstjens H, Rikxoort EM, Slebos DJ. Endobronchial valves for emphysema without interlobar collateral ventilation. N Engl J Med 2015; 373 (24):2325-35.

18. Trudzinski FC, Höink AJ, Leppert D, Fähndrich S, Wilkens $H$, Graeter TP, et al. Endoscopic lung volume reduction using endobronchial valves in patients with severe emphysema and very low FEV 1. Respiration 2016; 92(4):258-65. doi.org/10.1159/000448761.

19. Darwiche K, Karpf-Wissel R, Eisenmann S, Aigner C, Welter $\mathrm{S}$, Zarogoulidis $\mathrm{P}$, et al. Bronchoscopic lung volume reduction with endobronchial valves in Low-FEV 1 patients. Respiration 2016; 92(6):414-9. doi: 10.1159/000452629.

20. Simon M, Harbaum L, Oqueka T, Kluge S, Klose H. Endoscopic lung volume reduction coil treatment in patients with very low FEV1: an observational study. Ther Adv Respir Dis 2019; 97:444-50. doi: 10.1177/1753466618760133. 